\title{
Quasibound states at thresholds in multichannel impurity scattering
}

\author{
Sang Wook Kim, Hwa-Kyun Park, H-S Sim and Henning Schomerus \\ Max-Planck-Institut für Physik komplexer Systeme, Nöthnitzer Str 38, D-01187 Dresden, \\ Germany
}

Received 7 October 2002

Published 22 January 2003

Online at stacks.iop.org/JPhysA/36/1299

\begin{abstract}
We investigate the threshold behaviour of transmission resonances and quasibound states in the multichannel scattering problems of a one-dimensional (1D) time-dependent impurity potential, and the related problem of a single impurity in a quasi-1D wire. It was claimed before in the literature that a quasibound state disappears when a transmission zero collides with the subband boundary. However, the transmission line shape, the Friedel sum rule, and the delay time show that the quasibound states still survive and affect the physical quantities. We discuss the relation between threshold behaviour of transmission resonances, and quasibound states and their boundary conditions in the general context of multichannel scatterings.
\end{abstract}

PACS numbers: 73.23.-b, 03.65.Ge, 73.50.Bk

\section{Introduction}

Electronic transport properties in quasi-one-dimensional wires have gained much attention, not only due to scientific interest but also due to practical applications [1]. When an electron is restricted to a wire, confinement subbands are formed due to quantization of the transverse momentum. In clean straight wires without impurities the modes of the subbands decouple, which results in a perfectly quantized conductance. However, the modes (including evanescent ones) mix when impurities are introduced into the wire, and the transport properties show a rich and non-trivial behaviour. Detailed aspects of the mixing have been extensively investigated [2-7]. The impurities put into the wire usually give rise to bound states, which can be separated into two categories: true bound states with real energy and quasibound states with complex energy. The imaginary part determines the decay rate of the quasibound state.

Even in simple one-dimensional (1D) scattering problems quasibound states are quite common, for example resonant transmission states through a double barrier [8, 9] and Ramsauer-like resonances [10] in potential wells. According to the Breit-Wigner theory [11], for each quasibound state the transmission amplitude possesses a pole in the complex 
energy plane. In multichannel scattering problems another kind of quasibound states can appear; the Fano-type resonance originally proposed to explain auto-ionization in atomic physics [12]. When a discrete energy level interferes with a continuum of states, the excitation spectra become asymmetric. Each subband mode in a quasi-1D wire gives rise to a zero-pole pair of transmission in the complex energy plane, where the zero occurs on the real energy axis. As a consequence, there exist transmission zeros, and the zero-pole pairs lead to asymmetric resonance features [13].

Transport through a time-dependent (mostly periodically oscillating) potential is also a subject of increasing importance, with a growing number of applications [14-23]. One of the important features here is that an oscillating potential can transfer an incoming electron of energy $E$ with finite probability to 'Floquet' sidebands at $E \pm n \hbar \omega$, where $n$ is an integer and $\omega$ is the angular frequency of the oscillation. Even though this corresponds to an inelastic scattering, the process is coherent and non-dissipative. Hence, it can still be regarded as a multichannel scattering problem as in the quasi-1D case, with the sidebands being analogous to the subbands. However, from the physical point of view, the Fano-type quasibound states of the periodically oscillating impurity are somewhat different from those of quasi-1D system [24]. If a bound state exists due to an attractive static potential added to the oscillating potential, electrons in the incident channel can emit photons and drop to the bound state, and similarly electrons in the bound state can absorb photons and jump to the incident channel. A transmission resonance takes place when the energy difference between the incident channel and the bound state is equal to the energy of one or more photons of the oscillating potential. Even without bound states transmission resonances such as these can occur.

The multichannel subbands in the quasi-1D case and the Floquet sidebands in the oscillating potential case divide the complex energy plane into sub-domains with appropriate boundary conditions for the quasibound states. Upon varying an external parameter such as the strength of the impurity potential, the quasibound states change their location in complex energy plane. In the literature [20] it was claimed for the oscillating impurity that the quasibound state vanishes when the transmission zero collides with the sub-domain boundary and disappears. However, as we will demonstrate, the transmission line shape, the Friedel sum rule, and the delay time show that the quasibound states do not disappear abruptly. This clarifies the relation between threshold behaviour of transmission resonances, and quasibound states and their boundary conditions in the general context of multichannel scatterings.

In section 2, we briefly summarize our findings about the transmission resonances and quasibound states. In section 3, we investigate the characteristics of quasibound states in the scattering problem with a 1D oscillating delta-function impurity, and discuss Friedel sum rule and Wigner delay time. In section 4, we study our second example, the scattering from a static delta-function impurity in a quasi-1D wire. Finally, we conclude our paper in section 5.

\section{Boundary conditions of quasibound states in multichannel scattering}

In this section we summarize the main finding of this paper, which will be further illustrated in sections 3 and 4 . The transmission coefficient $t_{m n}(E)$ relates the incoming wave $\psi_{n}^{i n}$ to the scattered wave $\psi_{m}^{s c}$ by

$$
\psi_{m}^{s c}(E)=t_{m n}(E) \psi_{n}^{i n} .
$$

The resonance energies are found by locating the poles of $t_{m n}(E)$, namely

$$
\frac{1}{t_{m n}\left(E_{R}-\mathrm{i} E_{I}\right)}=0 \text {. }
$$


The poles of $t_{m n}(E)$ occur at complex energies $E=E_{R}-\mathrm{i} E_{I}$. At these complex energies, a scattered wave can be produced even if no incident wave is present. When we choose the sign convention of time as $\exp (-\mathrm{i} E t / \hbar), E_{I}$ must be positive for the system to be stable. In the Breit-Wigner case [11] the transmission near the quasibound state is given by $t(E) \sim 1 /\left[E-\left(E_{R}-\mathrm{i} E_{I}\right)\right]$, where the pole in complex energy plane completely determines the transmission line shape.

The poles can also be found by solving the wave equation with the appropriate boundary conditions of only outgoing waves. This can be described by the following linear equation

$$
M\left(\begin{array}{c}
\psi_{\text {out }}^{l} \\
\psi_{\text {out }}^{r}
\end{array}\right)=0
$$

Here $M$ is the matrix of wave-matching conditions, and $\psi_{\text {out }}^{l}$ and $\psi_{\text {out }}^{r}$ are the left and the right outgoing wave vectors, respectively. The matrix $M$ contains the wave number $k$ as a function of energy $E$. For real $E$ the wave number $k$ is given by $k=\sqrt{2 \mu E} / \hbar$, where $\mu$ is the mass. For complex energies, we have to choose the sign of the square root carefully. Considering the general relation [25]

$$
\sqrt{\alpha+\mathrm{i} \beta}= \pm\left(\sqrt{\frac{\alpha+\sqrt{\alpha^{2}+\beta^{2}}}{2}}+\mathrm{i} \frac{\beta}{|\beta|} \sqrt{\frac{-\alpha+\sqrt{\alpha^{2}+\beta^{2}}}{2}}\right)
$$

provided that $\beta \neq 0$, we obtain

$$
\sqrt{E_{R}-\mathrm{i} E_{I}}= \pm\left(k_{R}-\mathrm{i} k_{I}\right)
$$

where by definition $E_{I}, k_{R}$ and $k_{I}$ are positive. The real energy $E_{R}>0$ for propagating states, and $E_{R}<0$ for evanescent states. The correct choice of the sign in equation (5) depends on the analytical continuation based on the physical constraints on the real energy axis. For $E_{I}=0$ the following relation should be satisfied,

$$
\sqrt{E_{R}}= \begin{cases}k_{R} & E_{R}>0 \\ \mathrm{i} k_{I} & E_{R}<0\end{cases}
$$

where both $k_{R}$ and $k_{I}$ are still positive. The choice of sign for propagating states corresponds to the choice of the negative sign for $k_{I}$ (i.e. the positive sign for $k_{R}$ ), and follows from the propagating direction of the plane wave far from the scatterer. For evanescent states, the positive sign for $k_{I}$ must be chosen in order to avoid divergence of the solution in space.

It is worth noting that the ' + ' sign corresponds to the decaying wave $\exp \left(\mathrm{i} k_{R} x-\right.$ $\left.k_{I} x\right) \exp \left(-\mathrm{i} E_{R} t / \hbar-E_{I} t / \hbar\right)$ while the '-' sign corresponds to the diverging wave $\exp \left(\mathrm{i} k_{R} x+\right.$ $\left.k_{I} x\right) \exp \left(-\mathrm{i} E_{R} t / \hbar-E_{I} t / \hbar\right)$, with $x>0$. The physical reason of the exponential divergence of the channels with positive real energy is a retardation effect; the wave at $x \gg 1$ has propagated away from the impurity where it originated at a time $\Delta t \approx x / v$ in the past, where $v$ is the velocity of the wave. However, at that earlier time the amplitude of the wave at the impurity was larger by a factor $\exp \left(E_{I} \Delta t / \hbar\right)$. This corresponds to $\exp \left(k_{I} x\right)$ since $\left(E_{R}-\mathrm{i} E_{I}\right) / \hbar \simeq v\left(k_{R}-\mathrm{i} k_{I}\right) / 2$ [26].

For multichannel scattering, the wave numbers are given by

$$
k_{n}= \pm \sqrt{E_{R}-E_{n}-\mathrm{i} E_{I}}
$$

where $E_{n}$ represents the quantized energy of a transverse mode in the quasi-1D case ( $n=$ $1,2,3, \ldots)$ and is equal to $n \hbar \omega$ in the $1 \mathrm{D}$ oscillating potential case $(n=\ldots,-1,0,1, \ldots)$. We order the $E_{n}$ by magnitude and denote by $n^{*}$ the special value of $n$ which satisfies $E_{n^{*}}<E_{R}<E_{n^{*}+1}$. 
(a)
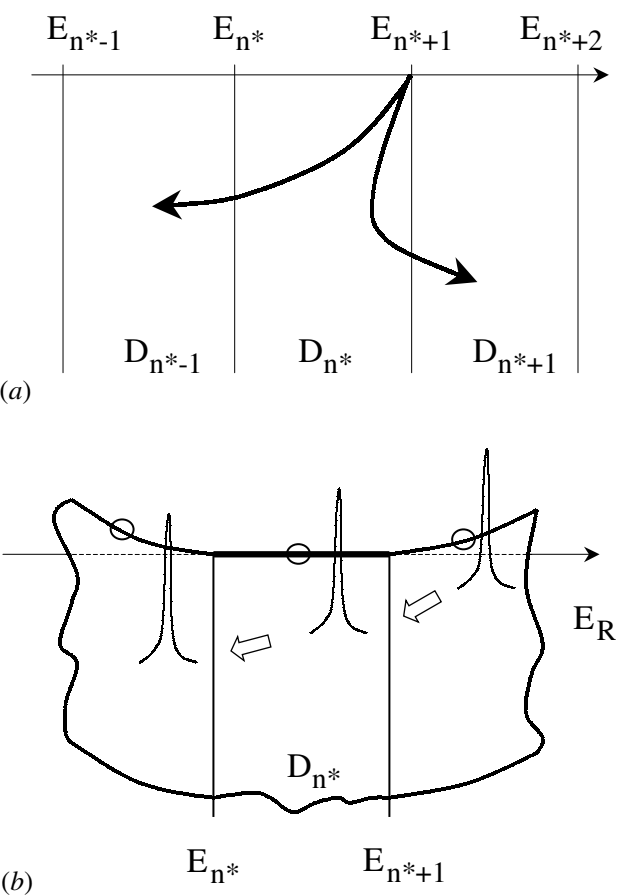

Figure 1. (a) Schematic diagram for possible motion of quasibound states in the complex energy plane. $E_{n}$ and $D_{n}$ denote subband threshold energies and domains, respectively. (b) Schematic of the proper Riemann surface $R$ of the domain $D_{n}$ in the complex energy plane. The circles and the peaks represent the transmission zeros and the quasibound states (or poles), respectively.

In order to satisfy the physical conditions for the signs of $k_{n}$ mentioned above, for $n \leqslant n^{*}$ the '-' sign should be chosen for Im $k_{n}$, and for $n>n^{*}$ the ' + ' sign, which corresponds to the selection of a certain Riemann sheet for evaluating the complex square root function. This choice of the signs will be called proper Riemann sheet since this set of signs corresponds to the correct analytical continuation from the real energy axis. In order to represent the Riemann sheet through the signs of Im $k$ such as in our convention, the square root functions should have their branch cuts on the real axis starting from the branch points and extending to the positive infinity. In general, the notion Riemann sheet depends on the choice of a branch cut. However, this choice of the branch cut cannot affect physical results, because it is only a way to label the projection of the Riemann sheets onto the complex plane.

We introduce a labelling for the Riemann sheets by the vector of signs of the square root function. In this notation the proper Riemann sheet is represented by $(\ldots,-,-, \ominus,+,+, \ldots)$, which implies ' -' for $\operatorname{Im} k_{n^{*}-2}$, '-' for $\operatorname{Im} k_{n^{*}-1}$, '-' for $\operatorname{Im} k_{n^{*}}$, '+' for $\operatorname{Im} k_{n^{*}+1}$, and '+' for $\operatorname{Im} k_{n^{*}+2}$, and $\ominus$ has been used to indicate the sign of $n^{*}$. We also call the regions of complex energies separated by the quantized energies $E_{n}$ 'domains', e.g. $D_{n}$ represents the region with $E_{n}<E_{R}<E_{n+1}$ as shown in figure 1(a).

As a parameter of the scattering potential such as its strength is varied, the locations of the poles change continuously and sometimes can collide with the boundary of the initial domain. We can trace the pole into the neighbouring domain through the boundary, for example from $D_{n^{*}}$ to $D_{n^{*}-1}$ in figure $1(a)$. In fact, the trajectory of the pole across the boundary is continuous and smooth once we use the same Riemann sheet as that used in $D_{n^{*}}$, 
i.e. $(\ldots,-,-, \ominus,+,+, \ldots)$. However, this does not correspond to the proper Riemann sheet $(\ldots,-,-, \oplus,+,+, \ldots)$ in this new domain $D_{n^{*}-1}$ since the inequality $E_{n^{*}}<E_{R}$ is violated. Thus, the solution obtained in $D_{n^{*}-1}$ using the proper Riemann sheet of $D_{n^{*}}$ does not have any physical meaning in the domain $D_{n^{*}-1}$ (more precisely on the real axis of $D_{n^{*}-1}$ ), but still can influence physics on the real axis of $D_{n^{*}}$, especially when the resonance is broad. Let us emphasize that only the branch points, $E_{n}$, play an important role in the threshold behaviour of transmission resonances, but not the placement of the cuts. Physics is in the choice of signs on the real energy axis, equation (5). This situation is summarized schematically in figure $1(b)$, which shows that only small part of the proper Riemann sheet of $D_{n^{*}}$, i.e. $E_{n^{*}}<E_{R}<E_{n^{*}+1}$, is attached to the real energy axis. Since in either $D_{n^{*}-1}$ or $D_{n^{*}+1}$ the quasibound state can survive on the proper Riemann sheet of $D_{n^{*}}$, it can still affect the physics in the region $E_{n^{*}}<E_{R}<E_{n^{*}+1}$, but has no effect on other parts of real axis. In the following sections we discuss two examples.

\section{1D delta-function impurity with time dependence}

As the first example we investigate a 1D scattering problem with a delta-function impurity oscillating at frequency $\omega$. This problem was studied before by Bagwell and Lake [4], who calculated electron transmission and discussed transmission resonances. Recently, Martinez and Reichl [20] have investigated this problem and found the existence of nonresonant bands in the transmission amplitude as a function of the strength of the potential and driving frequency. They observed a periodic behaviour of the conductance as a function of the scattering strength and of the oscillation frequency of the scatterer.

\subsection{Scattering matrix formulation}

The system is described by the Hamiltonian

$$
H(x, t)=-\frac{\hbar^{2}}{2 \mu} \frac{\mathrm{d}^{2}}{\mathrm{~d} x^{2}}+\left[V_{s}+V_{d} \cos (\omega t)\right] \delta(x)
$$

where the $\mu$ is the mass of an incident particle, and $V_{s}$ and $V_{d}$ represent the strength of the static and the oscillating potential, respectively. Using the Floquet formalism [27] the solution of this Hamiltonian can be expressed as

$$
\Psi_{E_{F l}}(x, t)=\mathrm{e}^{-\mathrm{i} E_{F l} t / \hbar} \sum_{n=-\infty}^{\infty} \psi_{n}(x) \mathrm{e}^{-\mathrm{i} n \omega t}
$$

where $E_{F l}$ is the Floquet energy which takes continuous values in the interval $0<E_{F l} \leqslant \hbar \omega$.

Since the potential is zero everywhere except at $x=0, \psi_{n}(x)$ is given by the following form,

$$
\psi_{n}(x)= \begin{cases}A_{n} \mathrm{e}^{\mathrm{i} k_{n} x}+B_{n} \mathrm{e}^{-\mathrm{i} k_{n} x} & x<0 \\ C_{n} \mathrm{e}^{\mathrm{i} k_{n} x}+D_{n} \mathrm{e}^{-\mathrm{i} k_{n} x} & x>0\end{cases}
$$

where $k_{n}=\sqrt{2 \mu\left(E_{F l}+n \hbar \omega\right)} / \hbar$. The wavefunction $\Psi_{E_{F l}}(x, t)$ is continuous at $x=0$,

$$
A_{n}+B_{n}=C_{n}+D_{n}
$$

and the derivative jumps by

$$
\left.\frac{\mathrm{d} \Psi_{E_{F l}}}{\mathrm{~d} x}\right|_{x=0^{+}}-\left.\frac{\mathrm{d} \Psi_{E_{F l}}}{\mathrm{~d} x}\right|_{x=0^{-}}=\frac{2 m}{\hbar^{2}}\left[V_{s}+V_{d} \cos (\omega t)\right] \Psi_{E_{F l}}(0, t) .
$$


Using equation (9) this leads to the condition

$$
\begin{aligned}
\mathrm{i} k_{n}\left(C_{n}-D_{n}-A_{n}+B_{n}\right) & =\gamma_{s}\left(A_{n}+B_{n}\right)+\gamma_{d}\left(A_{n+1}+A_{n-1}+B_{n+1}+B_{n-1}\right) \\
& =\gamma_{s}\left(C_{n}+D_{n}\right)+\gamma_{d}\left(C_{n+1}+C_{n-1}+D_{n+1}+D_{n-1}\right)
\end{aligned}
$$

where $\gamma_{s}=2 \mu V_{s} / \hbar^{2}$ and $\gamma_{d}=\mu V_{d} / \hbar^{2}$. After some algebra we have the following equation from equations (11), (13) and (14),

$$
\left(\begin{array}{l}
\vec{B} \\
\vec{C}
\end{array}\right)=\left(\begin{array}{cc}
-(I+\Gamma)^{-1} \Gamma & (I+\Gamma)^{-1} \\
(I+\Gamma)^{-1} & -(I+\Gamma)^{-1} \Gamma
\end{array}\right)\left(\begin{array}{l}
\vec{A} \\
\vec{D}
\end{array}\right)
$$

where

$$
\Gamma=\left(\begin{array}{ccccc}
\ddots & \ddots & 0 & 0 & 0 \\
\gamma_{d} / \mathrm{i} k_{-1} & \gamma_{s} / \mathrm{i} k_{-1} & \gamma_{d} / \mathrm{i} k_{-1} & 0 & 0 \\
0 & \gamma_{d} / \mathrm{i} k_{0} & \gamma_{s} / \mathrm{i} k_{0} & \gamma_{d} / \mathrm{i} k_{0} & 0 \\
0 & 0 & \gamma_{d} / \mathrm{i} k_{1} & \gamma_{s} / \mathrm{i} k_{1} & \gamma_{d} / \mathrm{i} k_{1} \\
0 & 0 & 0 & \ddots & \ddots
\end{array}\right)
$$

and $I$ is an infinite-dimensional square identity matrix. Equation (15) can also be expressed in the form $\mid$ out $\rangle=M \mid$ in $\rangle$, where $M$ connects the input coefficients to the output coefficients including the associated evanescent Floquet sidebands. In order to construct the scattering matrix we multiply an identity to both sides, $K^{-1} K \mid$ out $\rangle=M K^{-1} K \mid$ in $\rangle$, where $K_{n m}=\sqrt{k_{n}} \delta_{n m}$. Then we have $\vec{J}_{\text {out }}=\bar{M} \vec{J}_{i n}$, where $\vec{J}$ represents the amplitude of probability flux and $\bar{M} \equiv K M K^{-1}$. It should be mentioned that $\bar{M}$ is not unitary due to the evanescent modes included.

If we keep only the propagating modes, we obtain the unitary scattering matrix $S$ [21, 24], which can be expressed in the following form,

$$
S=\left(\begin{array}{cccccc}
r_{00} & r_{01} & \cdots & t_{00}^{\prime} & t_{01}^{\prime} & \cdots \\
r_{10} & r_{11} & \cdots & t_{10}^{\prime} & t_{11}^{\prime} & \cdots \\
\vdots & \vdots & \ddots & \vdots & \vdots & \ddots \\
t_{00} & t_{01} & \cdots & r_{00}^{\prime} & r_{01}^{\prime} & \cdots \\
t_{10} & t_{11} & \cdots & r_{10}^{\prime} & r_{11}^{\prime} & \cdots \\
\vdots & \vdots & \ddots & \vdots & \vdots & \ddots
\end{array}\right)
$$

where $r_{n m}$ and $t_{n m}$ are the reflection and the transmission amplitudes, respectively, for modes incident from the left; $r_{n m}^{\prime}$ and $t_{n m}^{\prime}$ are similar quantities for modes incident from the right. From $S$ we can obtain the total transmission coefficient for the propagating mode entering in the $m$ th channel,

$$
T_{m}\left(E_{F l}\right)=\sum_{n=0}^{\infty}\left|t_{n m}\right|^{2} .
$$

Figure 2 shows the transmission coefficient $T$ as a function of $\varepsilon$ for various values of a dimensionless potential strength $a_{d}$, where $\varepsilon=E / \hbar \omega$ ( $E$ is the kinetic energy of the incident particle) and $a_{d}=\mu V_{d}^{2} / 8 \hbar^{3} \omega$. We exploit the relation $T(E)=T_{m}\left(E_{F l}\right)$ where $E=E_{F l}+m \hbar \omega$. For small $a_{d}$ the transmission coefficient shows some signatures of Fanotype transmission resonance structures (figures $2(a)$ and $(b)$ ) whereas the resonance structure 


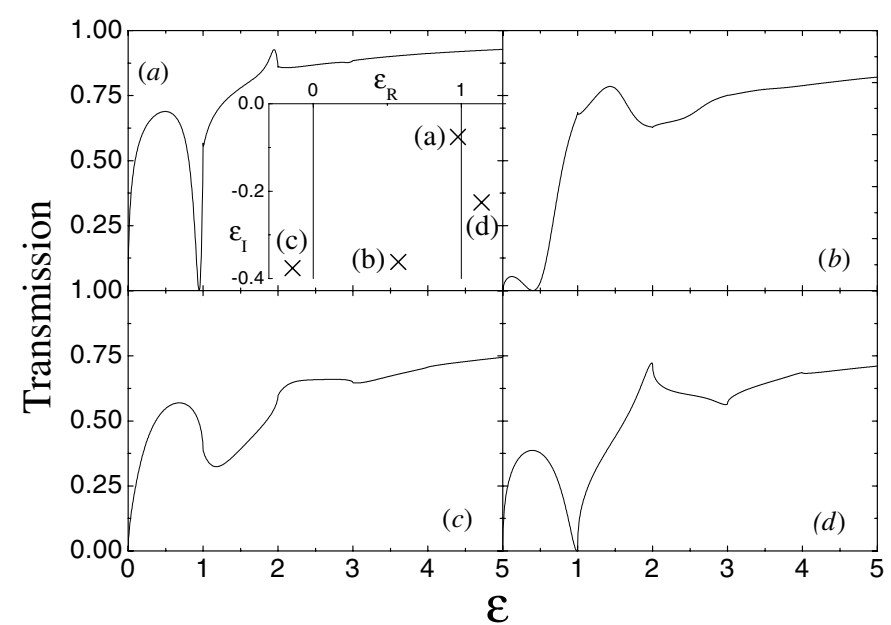

Figure 2. Transmission through an oscillating potential for $(a) a_{d}=0.2,(b) a_{d}=0.6$, (c) $a_{d}=1.0$ and $(d) a_{d}=1.2$ with $a_{s}=0$, where $a_{d}$ and $a_{s}$ represent the strength of the oscillating potential and the static part, respectively (for exact definitions see the text). The inset in (a) shows the locations of the poles in the complex energy plane for each case.

and transmission zero disappear as we increase $a_{d}$ (figure 2(c)). Although there exists no transmission zero in figure $2(c)$, the resonance-like structure (the peak around $\varepsilon=0.8$ ) still remains. For much larger $a_{d}$ the transmission zero reappears from $\varepsilon=1$. Let us note that the transmission coefficient has no symmetry of energy translation by $m \hbar \omega$ since in general $T_{m}\left(E_{F l}\right) \neq T_{m^{\prime}}\left(E_{F l}\right)$ when $m \neq m^{\prime}$.

\subsection{Quasibound states}

In order to obtain quasibound states we apply the same procedure used in the previous subsection, but with the different boundary conditions, $A=0$ and $D=0$. Since $B_{n}=C_{n}$ because of the reflection symmetry at $x=0$, after little algebra we obtain the linear equation

$$
\left(\begin{array}{ccccc}
\ddots & \ddots & 0 & 0 & 0 \\
\mathrm{i} \sqrt{a_{d}} & \mathrm{i} \sqrt{a_{s}}+\sqrt{\varepsilon-1} & \mathrm{i} \sqrt{a_{d}} & 0 & 0 \\
0 & \mathrm{i} \sqrt{a_{d}} & \mathrm{i} \sqrt{a_{s}}+\sqrt{\varepsilon} & \mathrm{i} \sqrt{a_{d}} & 0 \\
0 & 0 & \mathrm{i} \sqrt{a_{d}} & \mathrm{i} \sqrt{a_{s}}+\sqrt{\varepsilon+1} & \mathrm{i} \sqrt{a_{d}} \\
0 & 0 & 0 & \ddots & \ddots
\end{array}\right) \vec{C}=\left(\begin{array}{c}
\vdots \\
0 \\
0 \\
0 \\
\vdots
\end{array}\right) .
$$

Here $a_{s}=\mu V_{s}^{2} / 2 \hbar^{3} \omega$. We solve equation (19) for given $a_{s}$ and $a_{d}$ by using singular value decomposition [28] and find the complex energies of the quasibound states.

Due to the structure of the matrix in equation (19), if $\varepsilon^{\prime}$ is a solution of equation (19), then $\varepsilon^{\prime}+n$ is also a solution, where $n$ is an integer. We limit ourselves to the energy range of $0<\varepsilon_{R} \leqslant 1$, which means that the poles are meaningful only in the domain $D_{0}$. This constraint determines the signs of the complex square root functions in the diagonal of the matrix in equation (19) as described in section 2. Using the same notation as that used in section 2 the signs can be expressed by $(\ldots,+,+, \ominus,-,-, \ldots)$.

Figure 3(a) shows a trajectory of the quasibound state as $a_{d}$ is varied with $a_{s}=0$. The quasibound state of the first branch from the top originates from $(1,0)$ at $a_{d}=0$, moves 


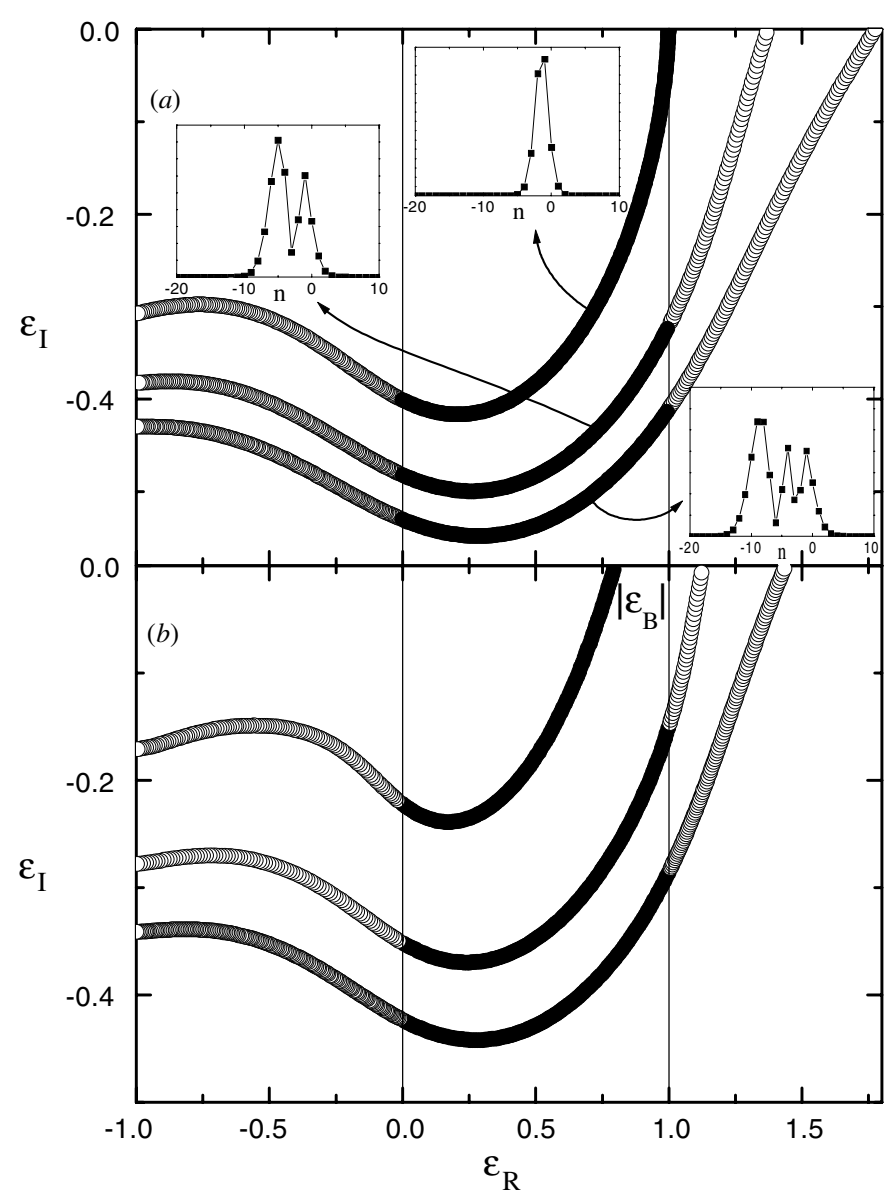

Figure 3. (a) Trajectory of a quasibound state (the full circles) as $a_{d}$ increases. The open circles represent the trajectory of the quasibound state continued into the neighbouring domains on the proper Riemann sheet of $D_{0}$. The quasibound state of the third branch from the top appears from the real axis at $a_{d} \simeq 1.869$, and collides with $\varepsilon_{R}=1$ and 0 at $a_{d} \simeq 2.325$ and 2.935, respectively (see the text for the first and the second branches). Insets: absolute squares of the solution vectors $\vec{C}$ of equation (19) at $a_{d}=0.5,1.5$ and 2.5 from the top, respectively. (b) Trajectory of quasibound states as $a_{d}$ increases $\left(a_{s}=-0.2\right)$. At $a_{d}=0$ the quasibound state is located at $\varepsilon=0.8$ due to the existence of a bound state, with binding energy $\varepsilon_{B}=0.2$. The quasibound state collides with $\varepsilon_{R}=0$ at $a_{d} \simeq 0.46$. A second branch of quasibound states appears from the real axis at $a_{d} \simeq 0.525$, and collides with $\varepsilon_{R}=1$ and 0 at $a_{d} \simeq 0.645$ and 1.22 , respectively. Finally, a third branch appears from the real axis at $a_{d} \simeq 1.221$, and collides with $\varepsilon_{R}=1$ and 0 at $a_{d} \simeq 1.475$ and 2.035 , respectively.

towards the domain $D_{-1}$, and crosses the domain boundary $\varepsilon_{R}=0$ at $a_{d} \simeq 0.935$. The trajectory of the pole helps to understand the transmission curves in figure 2 . The resonance structure of the transmission follows the pole, as is shown in figure 2 and the inset of figure 2(a). Since in the system considered here the transmission zero always takes place on the left of the pole in the real energy direction, it collides with the domain boundary $\varepsilon_{R}=1$ prior to the pole and disappears first at $a_{d} \simeq 0.782$. In [20] it is concluded that the quasibound state vanishes when the transmission zero disappears. Even after the pole moves out of the domain $D_{0}$ it still affects the transmission line shape, as shown in figure 2(c). There is no abrupt change of physical quantities, e.g. the transmissions, the 
Friedel phases and the Wigner delay time (these two will be shown below). The transmission zero disappears when it collides with the point $(0,0)$. We can trace the transmission zero even into the non-physical region $\varepsilon_{R}<0$ by calculating $S$ matrix on the proper Riemann sheet of $D_{0}$, although the unitarity of the $S$ matrix is violated.

A quasibound state of a second branch appears from the real axis at $a_{d} \simeq 1.032$ and moves to the left. It passes through the domain boundaries $\varepsilon_{R}=1$ at $a_{d} \simeq 1.31$, while the transmission zero appears in advance at $a_{d} \simeq 1.165$ as shown in figure $2(d)$. The pole finally leaves the domain $D_{0}$ by passing $\varepsilon_{R}=0$ at $a_{d} \simeq 1.935$. The second branch of the pole exactly corresponds to the case of the schematic shown in figure 1(b). In fact, one can find more branches of quasibound states in a regular fashion [20]. It is noted, however, that the branches qualitatively differ in their solution vectors, i.e. the null space of the matrix of equation (19) at the energy of quasibound state as shown in the insets of figure 3(a).

Figure $3(b)$ shows the trajectory of the pole in the case of a delta-function impurity with an attractive static potential added to the oscillating potential. A similar behaviour as in figure 3(a) is observed. The shifted starting point from $\varepsilon=1$ at $a_{d}=0$ is ascribed to the existence of the true bound state of an attractive delta-function potential with binding energy $E_{B}=-\mu V_{s}^{2} / 2 \hbar^{2}$. The introduction of the static potential has no effect on the signs of the complex square root functions, i.e. the Riemann sheet, which is clear in equation (19).

An interesting difference takes place in the trajectory of the quasibound states in the oscillating delta-function impurity when the attractive static potential is present. For small $a_{d}$ the matrix in equation (19) can be approximated by a $3 \times 3$ matrix involving only $k_{-1}, k_{0}$ and $k_{1}$, which corresponds to single-photon processes (absorption and emission). Using the results of appendix B of [4] it can be seen that this $3 \times 3$ matrix is not enough for the case without a static potential to describe the shift along the real energy axis of the quasibound states as $a_{d}$ is increased. At least a $5 \times 5$ matrix is needed to include the shift, which implies two-photon process. The mathematical reason is the fact that the slope $\mathrm{d} \varepsilon_{I} /\left.\mathrm{d} \varepsilon_{R}\right|_{a_{d}=0}=\infty$ in figure $3(a)$. On the other hand, in the case with an attractive potential $3 \times 3$ matrix is enough to obtain a shift of the pole along the real energy axis, as shown in figure $3(b)$ because $\mathrm{d} \varepsilon_{I} /\left.\mathrm{d} \varepsilon_{R}\right|_{a_{d}=0}$ has a finite value. With an attractive potential, a bound state already exists, and the oscillating potential leads to an ac-Stark shift [29] of that energy level. Without a static potential, however, one photon is needed to generate a bound state, and additional photons are needed to produce the ac-Stark-like shift.

\subsection{Friedel sum rule}

The number $N_{B}$ of bound states below an energy $E_{f}$ (say, the Fermi energy) is related to the Friedel phase $\theta_{F}=\ln \operatorname{Det}(S) / 2 \mathrm{i}$ of the scattering matrix $S$ through the Friedel sum rule [30] (see also [31])

$$
N_{B}=\frac{1}{\pi} \theta_{F}\left(E_{f}\right)
$$

This implies that each bound state contributes $\pi$ to the Friedel phase. Let us note that the scattering matrix in equation (17) is given as a function of a Floquet energy $\varepsilon_{F l}\left(\equiv E_{F l} / \hbar \omega\right)$ and the same holds for the Friedel phase, i.e. it is only relevant for $0 \leqslant \varepsilon<1$. Figures $4(a)$ and $(b)$ show Friedel phases as a function of $\varepsilon_{F l}$ for $a_{d}=0.6$ (the pole is located in $D_{0}$ ) and $a_{d}=1.0$ (the pole is in $D_{-1}$ ), respectively, with $a_{s}=0$. No abrupt change of the Friedel phase is observed at the threshold $a_{d} \simeq 0.782$ of the transmission zero or when the pole crosses the domain boundary, $a_{d} \simeq 0.935$.

It is noted in figure 4(a) that the Friedel phase does not exhibit a clear $\pi$ shift but looks even similar to figure $4(b)$. We expect the Friedel phase to shift by $\pi$ near quasibound states. 


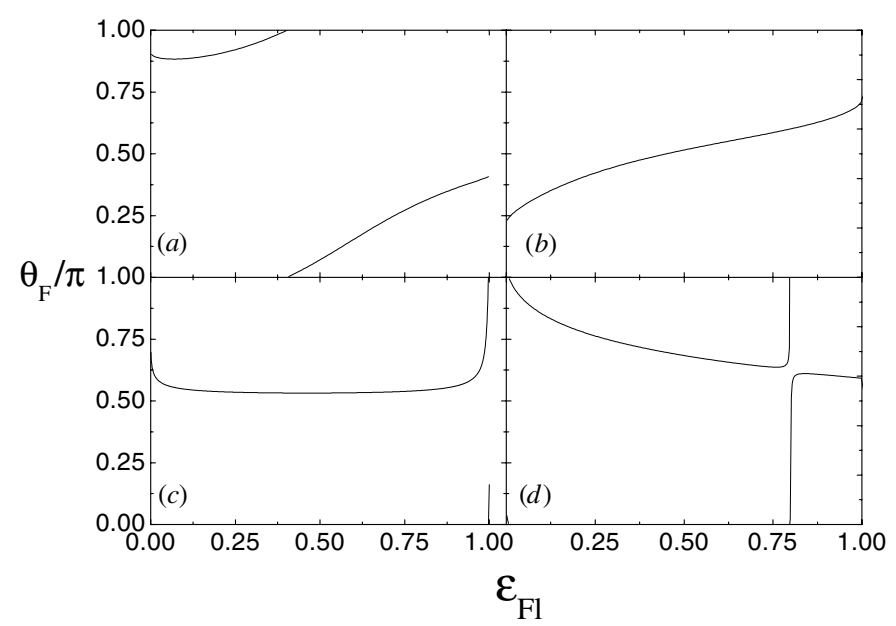

Figure 4. Friedel phases as a function of Floquet energy $\varepsilon_{F l}$ for $(a) a_{d}=0.6,(b) a_{d}=1.0$ and (c) $a_{d}=0.05\left(a_{s}=0\right)$. (d) $a_{d}=0.001$ with $a_{s}=-0.2$.

The absence of a clear $\pi$ shift in figure $4(a)$ is mainly ascribed to the large overlap between the resonance and the neighbouring domain since the imaginary part of the complex energy of a pole $\varepsilon_{I}$ is quite large, and so is the width of the resonance. Even though $\varepsilon_{I}$ is quite small for very small $a_{d}$, the overlap is still large enough to violate the $\pi$ phase shift by Friedel sum rule as shown in figure $4(c)$. The quasibound state is located quite close to the boundary $\varepsilon_{R}=1$ because of $\mathrm{d} \varepsilon_{I} /\left.\mathrm{d} \varepsilon_{R}\right|_{a_{d}=0}=\infty$. If we consider the oscillating potential with an additional attractive static potential, however, for very small $a_{d}$ a gap exists between the bound state and the domain boundary $\varepsilon_{R}=1$, which can make the overlap negligibly small, so that the phase shift $\pi$ is clearly seen in figure $4(d)$. When the Friedel sum rule is applied to the case of a multichannel scattering problem, the overlap between the resonance and neighbouring domains should be carefully considered.

\subsection{Wigner delay time}

The time scales associated with the quantum scattering have been a quite controversial issue (see e.g. [32]) because time is not a Hermitian operator in Hilbert space but a parameter. Nevertheless one can define the global time delay of wave packets by using a Hermitian time delay operator (see e.g. [33]). This time delay operator turns out to be related to the so-called Wigner-Smith time delay matrix [34], whose trace averaged over different channels gives a measure of the time delay of the scattered wave caused by the scattering potential (see also $[35,36])$. The Wigner delay time shows a peak at resonance energies, where the maximal value of the Wigner delay time approximately corresponds to the decay time $\hbar / \varepsilon_{I}$. To obtain the Wigner delay time we use the eigenvalues of the scattering matrix $S$. Due to the unitarity of $S$, all the eigenvalues lie on the unit circle and can be written in the form $\mathrm{e}^{\mathrm{i} \theta_{\alpha}}$. The Wigner delay time is defined by

$$
\tau_{W}=\hbar \sum_{\alpha} \frac{\mathrm{d} \theta_{\alpha}}{\mathrm{d} E}\left|\left\langle k_{n} \mid \theta_{\alpha}\right\rangle\right|^{2}
$$

where the eigenstate corresponding to the eigenvalue $\theta_{\alpha}$ and an input propagating state (or channel) with momentum $k_{n}$ are denoted by $\left|\theta_{\alpha}\right\rangle$ and $\left|k_{n}\right\rangle$, respectively [37]. It is worth noting 


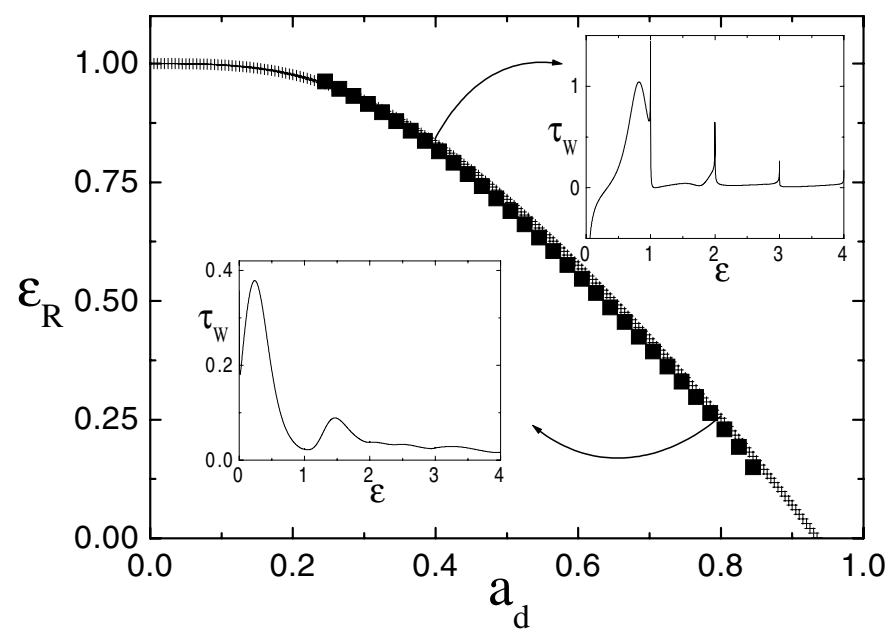

Figure 5. The real energies of the trajectory of a quasibound state (crosses) as a function of $a_{d}$ with $a_{s}=0$, compared to the energies of the local maxima of Wigner delay times (the full squares). The insets show Wigner delay times in unit of $1 / \omega$ as a function of energy $\varepsilon$ for $a_{d}=0.4$ (left) and $a_{d}=0.8$ (right).

that the Wigner delay time is a function of the energy of the incident particle. The insets of figure 5 show the Wigner delay time as a function of energy of an incident particle for two $a_{d}$ with $a_{s}=0$. The peaks of the Wigner delay time are located almost exactly at the real part of the complex energy of the pole in figure 5 (we omitted the data close to the domain boundaries where the Wigner delay time diverges), under the condition that the pole is located in $D_{0}$. No abrupt change of the Wigner delay time is again observed at the threshold of the transmission zero $\left(a_{d}=0.782\right)$. However, the peak disappears close to the domain boundary $D_{-1}$ since the pole enters the next domain.

\section{Delta-function impurity in quasi-1D wire}

As our second illustrative example, we study the simple quasi-1D scattering problem with an attractive static delta-function impurity of strength $|\gamma|$. In [3] it was found that dips in the transmission disappear as $|\gamma|$ is increased. Let us again discuss the relation to transmission poles.

\subsection{Scattering matrix formulation}

The Hamiltonian is given by

$$
H=\frac{p^{2}}{2 \mu}+\gamma \delta(x) \delta\left(y-y_{0}\right)+V_{c}(y)
$$

where $\gamma<0$, and $V_{c}$ represents a confinement potential which restricts the movement of the particle to the range $0<y<D$ in transversal direction. Using the complete set of the transversal modes $\chi(y)=\sqrt{2 / D} \sin (n \pi y / D)$, the solution of this Hamiltonian can be expressed as

$$
\psi_{E}(x, y)=\sum_{n=1}^{\infty} c_{n}(x) \chi(y) .
$$




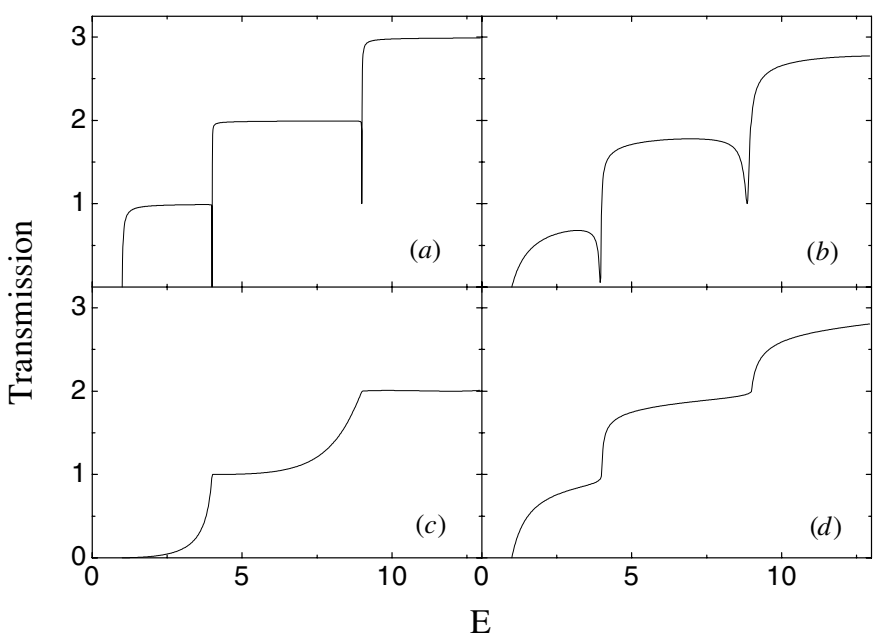

Figure 6. Transmission through a delta-function impurity in a quasi-1D wire with $(a) \gamma=$ $-2.28 \mathrm{feV} \mathrm{cm}^{2},(b) \gamma=-5.67 \mathrm{feV} \mathrm{cm}^{2},(c) \gamma=-9.1 \mathrm{feV} \mathrm{cm} \mathrm{cm}^{2}$ and $(d) \gamma=-22.75 \mathrm{feV} \mathrm{cm}$, where $\gamma$ represents the strength of the impurity potential.

Plugging this expansion into the Schrödinger equation and exploiting orthogonality of the transversal modes, we obtain the following equation

$$
\frac{\mathrm{d}^{2} c_{m}(x)}{\mathrm{d} x^{2}}+k_{m}^{2} c_{m}(x)=\sum_{n} M_{m n} c_{n}(x) \delta(x)
$$

where $M_{m n}=\left(4 \mu \gamma / D \hbar^{2}\right) \sin \left(m \pi y_{0} / D\right) \sin \left(n \pi y_{0} / D\right)$ and $k_{n}=\sqrt{2 \mu E / \hbar^{2}-(n \pi / D)^{2}}$. Using both the plane waves of equation (10) for $c_{n}(x)$ and the constraints in equations (11) and (12), we obtain the following conditions:

$$
\begin{aligned}
& A_{n}+B_{n}=C_{n}+D_{n} \\
& \mathrm{i} k_{n}\left(C_{n}-D_{n}\right)-\mathrm{i} k_{n}\left(A_{n}-B_{n}\right)=\sum_{m} M_{n m}\left(A_{m}+B_{m}\right) .
\end{aligned}
$$

These can be rewritten as

$$
\left(\begin{array}{l}
\vec{B} \\
\vec{C}
\end{array}\right)=\left(\begin{array}{cc}
(2 Q-M)^{-1} M & 2(2 Q-M)^{-1} Q \\
2(2 Q-M)^{-1} Q & (2 Q-M)^{-1} M
\end{array}\right)\left(\begin{array}{l}
\vec{A} \\
\vec{D}
\end{array}\right)
$$

where $Q_{m n}=\mathrm{i} k_{n} \delta_{m n}$. Following the same procedure as that used in equation (17) we can obtain the $S$ matrix for this problem and the total transmission coefficient

$$
T=\sum_{m n(\text { prop })}\left|t_{m n}\right|^{2}
$$

where the sum extends over all propagating modes. The resulting transmission coefficients are shown in figure 6 . We use the parameters $D=30 \mathrm{~nm}$ for the width of the wire, the mass $\mu=0.067 m_{e}$ as the effective mass of an electron in GaAs, $y_{0}=5 D / 12$ for the transversal position of the delta-function scatterer, and energy $E$ normalized by the $E_{1}=6.24 \mathrm{meV}$. The transmission coefficient depends on the number of the propagating channels, which will be discussed in the next subsection. 


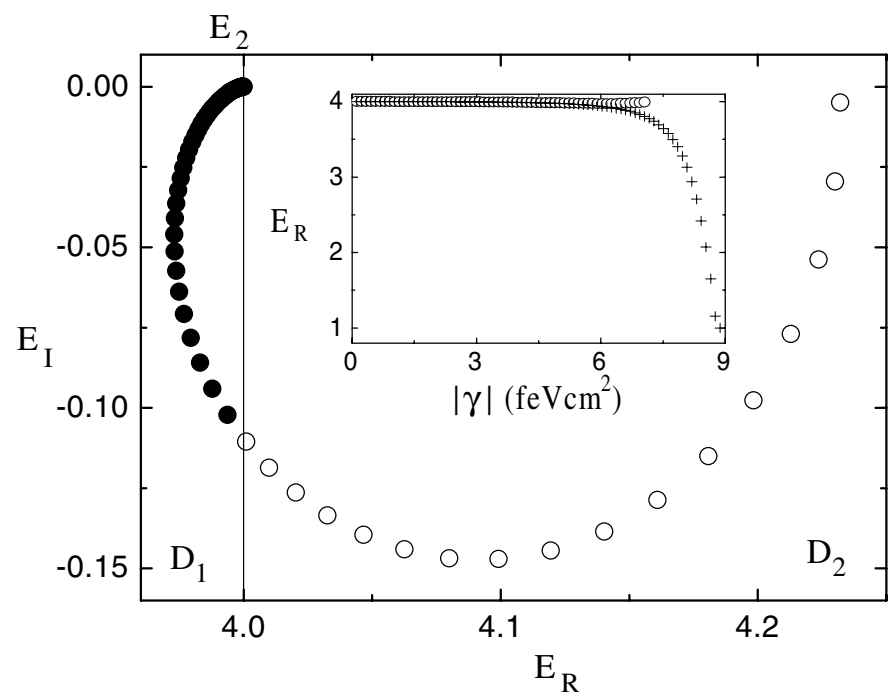

Figure 7. Trajectory of a quasibound state for a delta-function impurity in a quasi-1D wire (full circles) and its continuation into the neighbouring domain. Inset: real energies of the trajectory of a quasibound state (open circles) as a function of the strength of the delta-function impurity $(\gamma<0)$, and the energies of the transmission zeros (crosses).

\subsection{Quasibound states}

Quasibound states can be obtained from the following equation, derived from Green's function approach [38],

$$
\frac{1}{\gamma}-\sum_{n} \frac{4 \mu}{D \hbar^{2}}\left(\sin \frac{n \pi}{D} y_{0}\right)^{2} \frac{1}{2 \mathrm{i} k_{n}}=0
$$

which has to be solved for energy $E_{R}-\mathrm{i} E_{I}$ (entering via equation (7)). It should be mentioned that the location of the quasibound states and the transmission coefficients strongly depend on the number of modes $N_{c h}$ [38]. In fact they do not converge although $N_{c h}$ is increased. In order to model a realistic impurity one should take $N_{c h}$ large but finite, and fix it for all numerical calculations. $N_{c h}=100$ is chosen in this paper.

Figure 7 shows the trajectory of a quasibound state as $\gamma$ is varied. The quasibound state crosses the domain boundary $E_{2}$ at $\gamma \simeq-7.11 \mathrm{feV} \mathrm{cm}^{2}$, and enters the next domain $D_{2}$ (the open circles in figure 7) on the proper Riemann sheet of the domain $D_{1},(\ominus,+,+, \ldots)$. The situation is similar to that in section 3 except that the quasibound state collides with the real axis at $\gamma \simeq-9.06 \mathrm{feV} \mathrm{cm}^{2}$ and only one branch of quasibound states exists. As $|\gamma|$ increases the transmission zero moves far away from the pole, as is shown in the inset of figure 7 , and finally vanishes when it collides with the other domain boundary $E_{1}$ at $\gamma=$ $-8.83 \mathrm{feV} \mathrm{cm}^{2}$.

Apart from the quasibound state, the attractive delta-function impurity also accommodates one true bound state below $E_{1}$. Although the quasibound state vanishes at the threshold value of $\gamma$, the true bound state exists regardless of the value of $\gamma$, and its energy monotonically decreases as $|\gamma|$ increases. 


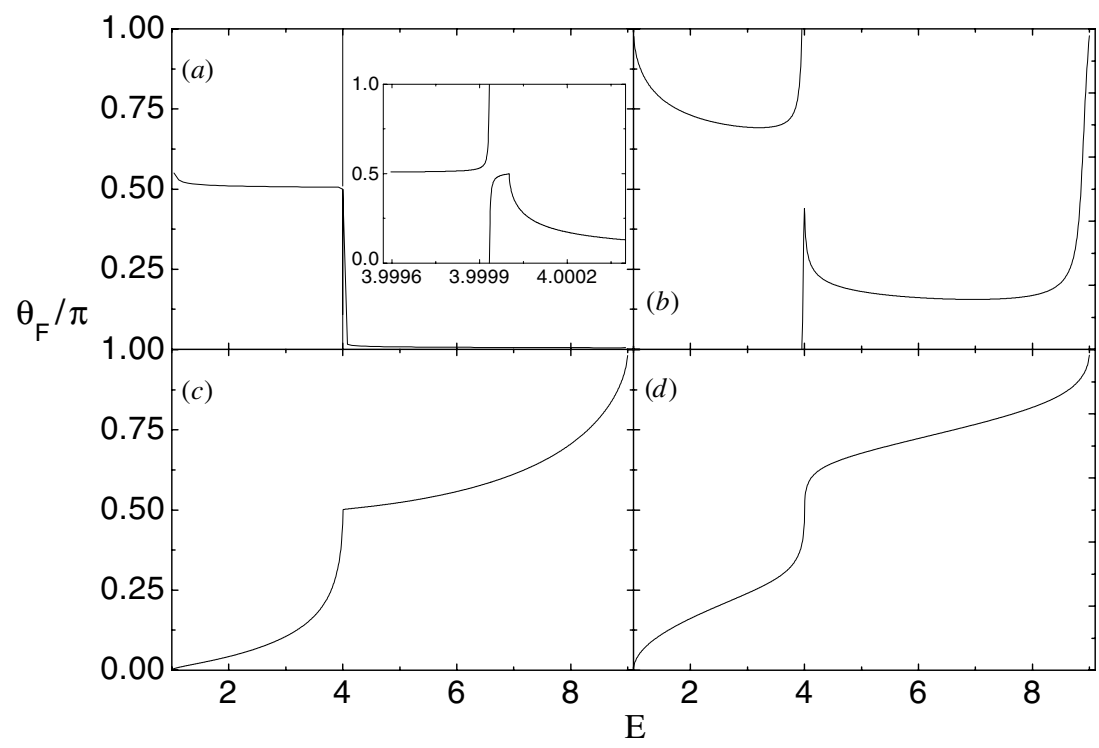

Figure 8. Friedel phases in the quasi-1D wire with a static impurity as a function of energy $E$ for (a) $\gamma=-0.57 \mathrm{feV} \mathrm{cm} \mathrm{cm}^{2}$, (b) $\gamma=-5.69 \mathrm{feV} \mathrm{cm}^{2}$, (c) $\gamma=-9.1 \mathrm{feV} \mathrm{cm} \mathrm{cm}^{2}$ and $(d) \gamma=$ $-11.38 \mathrm{feV} \mathrm{cm}^{2}$. The inset in panel $(a)$ shows the magnification of the resonance region.

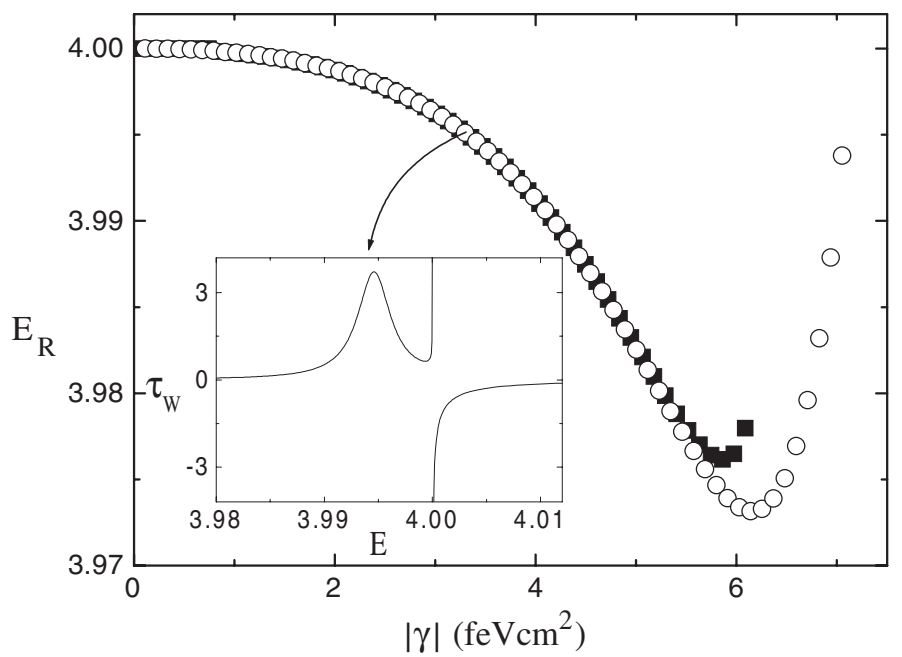

Figure 9. The real energies of the trajectory of a quasibound state (open circles) as a function of $|\gamma|$, compared to the energies of the local maxima of Wigner delay times (full squares). The inset shows Wigner delay time $\tau_{W}$ in units of $10^{-12} \mathrm{~s}$ as a function of energy $E$ for $\gamma=$ $-3.41 \mathrm{feV} \mathrm{cm}^{2}$, with the local maximum at $E=3.995$.

\subsection{Friedel sum rule and Wigner delay time}

The Friedel phase of the impurity in the quasi-1D wire is shown in figure 8 . The $\pi$ phase shift by quasibound state is clearly observed for very small $|\gamma|$, but it is hard to be resolved for larger $|\gamma|$ due to the overlap between the resonance and the neighbouring domain. Figure 9 shows 
the peaks of Wigner delay time and the real part of the complex energy of the pole, which coincide very well for $|\gamma| \leqslant 5.5 \mathrm{feV} \mathrm{cm}^{2}$. For larger $|\gamma|$ it is again difficult to distinguish the peaks of the Wigner delay time from the background which diverges at the domain boundary $E_{2}$ (see figure 7). As $|\gamma|$ increases the pole first moves away from $E_{2}$, and then moves back towards $E_{2}$. The peak of the Wigner delay time originating from the pole strongly overlaps with the divergent part of the background when it changes the direction. Hence, for large $|\gamma|$ the position of the peak no longer coincides with the energy of the pole. Friedel phase and Wigner delay time of the quasi-1D case behave similar to the case of an oscillating impurity.

\section{Summary}

We have investigated the characteristics of transmission resonances, Friedel phases and Wigner delay times in multichannel scattering problems focusing on the trajectories of quasibound states in the complex energy plane as a scattering strength is changed. Subband thresholds divide the complex energy plane into sub-domains characterized by appropriate boundary conditions for the quasibound states. This set of boundary conditions is related to the choice of the signs of the complex wave number $k_{n}$ in each subband. A transmission resonance (or a transmission zero) can vanish when it collides with the subband boundary, which, however, does not mean that the pole becomes completely irrelevant, as is seen in the transmission line shape, the Friedel phase and the delay time. Even if a quasibound state enters the neighbouring domain, it can still effect physics at the real energies of the domain from which it originated. We illustrate these findings with two simple examples: the scattering problem with the 1D oscillating delta-function impurity and the static delta-function impurity in quasi-1D wire.

\section{Acknowledgment}

We would like to thank Ingrid Rotter for a careful reading of our manuscript and helpful comments.

\section{References}

[1] Datta S 1995 Electronic Transport in Mesoscopic Systems (Cambridge: Cambridge University Press)

[2] Datta S, Cahay M and McLennan M 1987 Phys. Rev. B 36 R5655

Cahay M, McLennan M and Datta S 1988 Phys. Rev. B 3710125

[3] Bagwell P F 1990 Phys. Rev. B 4110354

[4] Bagwell P F and Lake R K 1992 Phys. Rev. B 4615329

[5] Levinson Y B, Lubin M I and Sukhorukov E V 1992 Phys. Rev. B 4511936

[6] Kunze C and Lenk R 1992 Solid State Commun. 84457

[7] Wang J, Zheng Q and Guo H 1997 Phys. Rev. B 559770

[8] Price P J 1988 Phys. Rev. B 381994

[9] Büttiker M 1988 IBM J. Res. Dev. 3263

[10] Ramsauer C 1921 Ann. Phys., Lpz 65513

Ramsauer C 1921 Ann. Phys., Lpz 66546

[11] Breit G and Wigner E 1936 Phys. Rev. 49519

[12] Fano U 1961 Phys. Rev. 1241866

[13] Porod W, Shao Z and Lent C S 1993 Phys. Rev. B 488495

[14] Büttiker M and Landauer R 1982 Phys. Rev. Lett. 491739

[15] Wagner M 1994 Phys. Rev. B 4916544

Wagner M 1995 Phys. Rev. A 51798

Wagner M 1996 Phys. Rev. Lett. 764010

Wagner M and Zwerger W 1997 Phys. Rev. B 55 R10217

Wagner M 1998 Phys. Rev. B 5711899 
[16] Sun Q F, Wang J and Lin T H 1998 Phys. Rev. B 5813007

[17] Burmeister G and Maschke K 1998 Phys. Rev. B 5713050

Burmeister G and Maschke K 1999 Phys. Rev. 594612

[18] Switkes M, Marcus C M, Campman K and Gossard A C 1999 Science 2831905

[19] Park H, Park J, Lim A K L, Anderson E H, Alivisatos A P and McEuen P L 2000 Nature 40757

[20] Martinez D F and Reichl L E 2001 Phys. Rev. B 64245315

[21] Henseler M, Dittrich T and Richter K 2001 Phys. Rev. E 64046218

[22] Moskalets M and Büttiker M 2002 Phys. Rev. B 66205320

[23] Kim S W 2002 Phys. Rev. B 66235304

[24] Li W and Reichl L E 1999 Phys. Rev. B 6015732

[25] Ahlfors L V 1979 Complex Analysis (New York: McGraw-Hill)

[26] Nöckel J U 1997 PhD Thesis Yale University

[27] See, for example, Reichl L E 1992 The Transition to Chaos in Conservative Classical Systems: Quantum Manifestations (New York: Springer)

[28] Press W H et al 1988 Numerical Recipes in C (Cambridge: Cambridge University Press)

[29] Bakos J S 1977 Phys. Rep. C 31209

[30] Friedel J 1958 Suppl. Nuovo Cimento 7287

[31] Harrison W A 1979 Solid State Theory (New York: Dover)

[32] Landauer R and Martin Th 1994 Rev. Mod. Phys. 66217

[33] Bracher C and Kleber M 1995 Ann. Phys., Lpz. 4696

[34] Wigner E P 1955 Phys. Rev. 98145

[35] Smith F 1960 Phys. Rev. 118349

[36] Fyodorov Y V and Sommers H-J 1997 J. Math. Phys. 381918

[37] Emmanouilidou A and Reichl L E 2002 Phys. Rev. A 65033405

[38] Boese D, Lischka M and Reichl L E 2000 Phys. Rev. B 615632 